\title{
Competencias ciudadanas y educación superior, una alternativa al desarrollo
}

\section{Ph.D Cabrera J. Manuel F. a , Msc Gómez-Reyes Flor Marleny b}

${ }^{a}$ Doctor en estudios políticos, Vicerrectoría de investigación, Magister en Educacion, Especilista y profesional en Comercio Internacional. Docente de la Facultad de ciencias económicas y administrativas Universidad ECCI (Colombia, mcacreraj@ecci.edu.co), ${ }^{b}$ Magister en Gestión de Organizaciones, Especialista en Estándares internacionales de Contabilidad y Auditoría, Contadora Pública y docente en la Universidad Cooperativa de Colombia (Colombia, fgomezr1@ucentral.edu.co).

\begin{abstract}
In the current Colombian context where it seeks to generate greater stability in the territory on a political and social level, after a long period of internal conflict, a historical opportunity is glimpsed through a peace agreement, in the process of gestation As part of the country's strategic process of transformation, which demands quality education as a basis for decreasing social inequality and strengthening social capital expressed in confidence and greater civility. In this sense, higher education and its correlation with civic competencies, the central object of this study, demands that the institutions raise and develop strategies that potencialicen in the citizen capacities that allow it to be consolidated as an agent Responsible for their own social change and that of their environment, promoting from the associativity a social fabric based on networks that fosters the trust and civic culture. Universities need to train new professionals based on curriculums strengthened with competences for the benefit of an inclusive and participatory society to strengthen democracy, promote peaceful solutions and socially established order, Analysis conducted as a case study, in the context of the community in ECCI .
\end{abstract}

Keywords: Higher Education. Social Development. Citizen Competitions

\section{Resumen}

En el actual contexto colombiano donde se busca generar una mayor estabilidad en el territorio a nivel político y social, después de un largo periodo de conflicto interno, se vislumbra una oportunidad histórica por medio de un acuerdo de paz, en pleno proceso de gestación como parte del proceso estratégico de transformación del pais, el cual demanda de educación de calidad como base para disminución de la desigualdad social y fortalecimiento del capital social expresado en confianza y mayor civilidad. En este sentido, la educación superior y su correlación con competencias ciudadanas, objeto central de este estudio, demanda que las instituciones planteen y desarrollen estrategias que potencialicen en el ciudadano capacidades que le permita se consolide como agente responsable de su propio 
cambio social y el de su entorno, fomentando desde la asociatividad un tejido social basado en redes que fomente la confianza y cultura civica. Las Universidades requieren formar nuevos profesionales con base en curriculos fortalecidos con competencias en beneficio de una sociedad incluyente y participativa para fortalecer la democracia, promover soluciones pacificas y el orden socialmente establecido, análisis realizado como estudio de caso, en el contexto de la comunidad en Universidad ECCI (Escuela Colombiana de Carreras industriales).

Palabras clave: Educación superior. Desarrollo social. Competencias ciudadanas.

\section{Introducción}

En el actual entorno colombiano en los últimos años los procesos dinámicos de negociación entre gobierno y grupos al margen de la ley han generado una nueva realidad denominada posconflicto. Los procesos demanda articulación y entendimiento para su materialización con el compromiso de todos los estamentos sociales a nivel público y sociedad civil. Después de un largo periodo de conflicto interno los objetivos deben ser comunes para potencializar la estabilidad en el territorio a nivel político y social.

En este contexto, el rol de la educación superior es altamente incidente, ya que desde su esfera permea el comportamiento cívico de la comunidad. El desarrollo de estrategias educativas potencializan en los ciudadanos capacidades colectivas que los consolidan como agentes responsables de sus propios cambios y el de su entorno. Las estrategias pedagógicas fomentan desde el trabajo en equipo y la asociatividad con diferentes agentes, redes que promueven el interés general sobre el particular. En tal sentido, las instituciones de educación superior le están apostando a currículos que no se centran exclusivamente en conocimientos disciplinares, sino que estos se articulen con habilidades que evidencien competencias que empoderen al ciudadano frente a responsabilidades y derechos cívicos. Las universidades aportan a la trasformación social del país a través del fortalecimiento de agentes generadores de cambio.

El estudio de las competencias ciudadanas en Colombia se remonta a trabajos realizados por el Ministerio de Educación Nacional (MEN), principalmente en educación primaria y básica. A partir del año 2004 el MEN en su programa de "Estándares básicos de competencias ciudadanas" definió las competencias como: "el conjunto de conocimientos y habilidades cognitivas, emocionales y comunicativas que, articulados entre sí, hacen posible que el ciudadano actúe de manera constructiva en la sociedad democrática" (Mieles \& Alvarado, 2012, pág. 64). [5]. En el sistema educativo colombiano los estudiantes de bachillerato se gradúan en una edar promedio de 15 a 17 años, por ello el promedio de la edad de ingreso a la universidad es de 16 años.

Abordar el análisis y la reflexión de las competencias ciudadanas es una tarea que demanda la articulación de conocimientos objetivos y subjetivos del ser social. En los contextos afectados por características exógenas del entorno y mediados por la incidencia de estructuras e instituciones el ciudadano interactúa en escenarios cotidianos que permean su 
tránsito de la esfera de lo individual a lo social. La educación y cultura del ciudadano expresa sus comportamientos sociales que son validados por el colectivo, denominado también, habitus (Bourdieu, 2000). Las experiencias individuales del sujeto, en cómo se articula conoce y reconoce los ambientes próximos son las características que transgreden su comportamiento expresado en su grado de participación cívica.

Ocuparse del estudio y evaluación de competencias ciudadanas como problema central, es sin duda una mediación para fortalecer la democracia. Con las competencias ciudadanas se promoven soluciones pacíficas que incrementar la tolerancia y fortalecer el respeto de las normas en un orden socialmente establecido. Lo anterior, privilegia el bien común y potencializa la vida cívica en una sociedad que requiere una transformación substancial en función del interés colectivo.

Para abordar esta problemática se plantea un método de investigación descriptivo con enfoque de análisis cuantitativo principalmente. Se investigará en torno al desarrollo y apropiación de competencias ciudadanas en la educación superior. El análisis se realizará en la comunidad académica de la universidad ECCI (Escuela Colombiana de Carreras Industriales) como estudio de caso. Se puede asumir como un referente de la tendencia de formación en competencias ciudadanas en educación superior en Colombia.

\section{Obejtivos}

Analizar la correlación entre educación superior y competencias ciudadanas en Colombia.

\subsection{Objetivos específicos}

\subsubsection{Identificar los referentes teóricos sobre competencias ciudadadas}

1.1.2. Explicar la concepción liberal de ciudadanía

\subsubsection{Categorizar las competencias ciudadanas en Colombia}

\section{Desarrollo de la innovación}

\subsection{Referentes Teóricos}

Para comprender el alcance del vínculo entre competencias ciudadanas y educación superior, se hace necesario plantear un acercamiento a la dinámica propia de la concepción y su articulación con educación en el contexto colombiano. Dicho campo comenzó a explorarse principalmente a finales del siglo XX, identificando inicialmente en el campo de la educación media y básica asumido como su principal campo de acción, lo que ha llevado en teoría a generar transformaciones con impacto social, que sin duda favorecen una educación coherente con las necesidades del país y la sociedad.

Este paradigma, sobre un nuevo enfoque de la educación con calidad en el país, ha fortalecido el desarrollo de transformaciones sociales en cierto grado pero no aun con el impacto esperado. Pues se puede observar que desde finales de los años noventa como resultado de la dinámica económica, política y social del territorio que ha sido impactada por 
un conflicto interno de grandes proporciones lo que ha generado problemáticas que se expresan en altos niveles de desigualdad y comportamiento violento de algunos ciudadanos, lo que desencadena problemas de convivencia y civilidad a nivel local, realidades complejizadas por la incidencia de cambios geopolíticos globales que afectan el territorio como por ejemplo los problemas migratorios de venezolanos al país, así como el problema de desplazamiento interno por el conflicto armado.

Para comprender este fenómeno se hace necesario asumir la ciudadanía como una categoría social, donde el sujeto es objeto de deberes y derechos en un conglomerado social estructurado con un orden político, La ciudadanía permite plantear y desarrollar acuerdos comunes por medio de la democracia y la participación cívica Kaldor, (2005) [4].

En este sentido, ejercer de forma armoniosa la ciudanía, conlleva enfrentar desafíos que surgen desde comprender diferencias a nivel económico, social, cultural, educativito y cognitivo que pueden generar anomalías entre su fin y su desarrollo en un contexto de sistema político estructurado, basado en normatividad y un sistema de educación que ampara las libertades individuales, en este sentido, "el ejercicio de la ciudadanía implica el reto de construir sociedad a partir de la diferencia, es decir, del hecho de que a pesar de que compartimos la misma naturaleza humana, somos diferentes en muchas maneras" (Chaux, Lleras, \& Velásquez, 2004, pág. 19) [2].

\subsubsection{Un acercamiento a la concepción liberal de ciudadanía}

Desde la perspectiva de un enfoque liberal planteado por Rawls (2006) [9] la ciudadanía es un constructo colectivo que articula elementos de la esferas privada del ciudadano con escenarios públicos moldeada por la cultura, lo que incide en las dinámicas propuestas del Estado-nación y como estas se relacionan con la sociedad, posibilitando y generando vínculos que facilitan un conglomerado incluyente a partir del reconocimiento y respeto de las libertades y los derechos.

En términos de Rawls (1995) [8] la ciudadanía es una característica individual del sujeto que parte del reconocimiento y valoración del bien de sus libertades, y como esta expresión logra armonizarse con la estructura socio política del territorio.

La ciudadanía se expresa a partir del reconocimiento moral propio de la facultad de la persona, Rawls (2006) [9] a partir de la valoración de la libertad expresada en que los sujetos son libres y así se auto conciben y a la vez, reconocen esta característica en las otras personas, de igual forma, tienen la capacidad de reconocer y validar reivindicaciones colectivas y asumen responsabilidades frente a sus objetivos, lo que incide en la esfera de lo público a partir de cómo se validan sus exigencias permeadas por la moral

Este enfoque de ciudadanía reconoce que la justicia es compartida y comprendida como un acuerdo público, basado en principios de asociatividad colectiva donde se asume esta, como una virtud destacable de las instituciones, que deben garantizar que sean justas y equitativas. "Por tanto, en una sociedad justa, las libertades de la igualdad de ciudadanía se dan por establecidas definitivamente; los derechos asegurados por la justicia no están sujetos a regateos políticos ni al cálculo de intereses sociales" (Rawls, 2006, pág. 17) [8].

Una sociedad que está en capacidad de construir vínculos de respeto y reconocimiento entre ciudadanos e instituciones, tiende a generar procesos de construcción democrática solidos que favorecen la interacción cívica del conglomerado expresada en una democracia 
más sólida y participativa, en este sentido, la educación cobra relevancia y se convierte en un medio eficiente para consolidar sociedades incluyentes y participativas.

\subsubsection{Competencias ciudadanas y educación en Colombia}

Cuando se habla de competencias ciudadanas en el entorno de la educación, se hace alusión al constructo de conocimientos y habilidades y comportamientos que expresa el sujeto en el marco de sus relaciones con el entorno social, planteado por el Ministerio de Educación Nacional en 2004 es decir, cuando su accionar traspasa de su esfera individual al entorno de interactividad colectiva, en su accionar ciudadano, el cual está moldeado por una estructura que permite la interacción entre organizaciones y sociedad civil. El Ministerio de Educación Nacional, parte reconocer una concepción de ciudadanía que da cuenta de su impacto en el desarrollo de la vida de las personas.

El concepto de ciudadanía que está en la base de los Estándares Básicos de Competencias Ciudadanas parte de la premisa básica de que es característica de los seres humanos vivir en sociedad. Las relaciones humanas son necesarias para sobrevivir y para darle sentido a la existencia. Desde el momento mismo de su nacimiento, niños y niñas empiezan a aprender a relacionarse con otras personas y a entender qué significa vivir en sociedad. Este aprendizaje continúa toda la vida (Ministerio de Educación Nacional, 2006, pág. 149) [7].

Esta concepción guarda concordancia con los principios de la Constitución Política de Colombia de 1991, que reconoce la diversidad de la población a nivel cultural, étnico y social lo que implica considerar la diferencia, y de esta forma potencializar la inclusión respetando las particularidades de la población. Sin embargo, en el desarrollo del ejercicio de la ciudadanía evidenciado en la cotidianidad de la interactividad social del país expresado en los niveles de violencia endógenos conocidos ampliamente, se perciben asimetrías que conllevan a violación de derechos o acciones de la comunidad que afectan el orden ciudadano segregando a algunos grupos o miembros de la sociedad y a la vez, se identifican comportamientos de algunos actores que con sus acciones empañan el sentir ciudadano priorizando el interés particular sobre el colectivo.

Frente a esta realidad, se hace evidente la prioridad de mejorar substancialmente la calidad de la educación en el país a partir del fomento de procesos de formación ciudadana que potencialicen las habilidades sociales de los habitantes, lo que redundará en mayor capital social acumulado, como lo plantean Woolkock \& Narayan (2001) [11]. Quienes, consideran este como un factor incidente en el desarrollo de una comunidad basado en la asociatividad y mayor participación en la vida cívica en función de privilegiar el interés comunitario.

El fomento de educación en competencias ciudadanas potencializa a mediano y largo plazo, estrategias basadas en la asociatividad entre instituciones y sociedad civil, lo que en términos de Robinson (2003) [10] disminuye la brecha en pobreza entre segmentos de la población marginales a partir de empoderarlos frente a sus mismas necesidades y capacidades fomentando así, emprendimientos sociales.

En tal sentido, la educación cobra un rol relevante en función del desarrollo social de una comunidad, una mejor formación en competencias ciudadanas redunda en mayor vida cívica, fortalecimiento de la democracia y mayor cohesión que permite disminuir los niveles de desigualdad.

Implícitamente las competencias ciudadanas conllevan un escenario moldeado por competencias comunicativas, cognitivas y transversales que viabilizan la interacción de los sujetos sociales a partir de reconocer en el otro un punto de vista valido que permite procesos de dialogo para generar vínculos y relaciones potencialmente equitativas, a partir de 
posibilitar el medio como de encontrar un punto de equilibrio entre las partes (Álvarez, Sandoval, \& Puello, 2018) [1].

El Ministerio de Educación Nacional desde el año 2004, ha planteado como principio de política pública transversal de calidad en la educación nacional, el desarrollo de la dimensión de competencias ciudadanas como un medio para trasformar la sociedad facilitando al ciudadano herramientas que permitan su interacción pacífica y aportar desde sus acciones individuales, a la construcción de una sociedad más respetuosa de la diferencia que propenda por el bienestar colectivo, en este sentido propone un programa denominado "Estándares básicos de competencias ciudadanas"

El programa se ha estructurado en tres grandes componentes, el primero de ellos orientado al fortalecimiento de la convivencia como camino a la construcción de paz, donde busca fortalecer el reconocimiento de las personas y el respeto de sus derechos humanos, en segundo lugar, el fortalecimiento de la democracia expresado en mayor participación lo que conlleva a un respeto por las normas, las leyes y la constitución en armonía con los derechos de los individuos que posibilitan la sociedad. Y finalmente, el respeto por la pluralidad, la cultura y la identidad que busca fortalecer el respeto por la diferencia y los derechos de los demás.

El programa de formación en competencias ciudadanas ha generado resultados positivos a nivel de convivencia. En relación con los resultados de 2002/03, los niños, niñas y jóvenes muestran mejores herramientas y competencias para hacer frente a los retos de la sociedad actual, empleando algunas de las estrategias que proporcionan diferentes áreas como las matemáticas, el lenguaje, las ciencias naturales y sociales, y las competencias ciudadanas, para analizar información y resolver los problemas cotidianos encontrados en sus clases, y para participar pacífica y constructivamente en las decisiones de la escuela, la familia y el entorno inmediato (MEN, 2006) [6].

Más allá de estos avances significativos, se ha demostrado que el programa se queda corto en relación con la necesidad de incrementar la equidad a nivel nacional, pues Colombia es un país con alto nivel de desigualdad, ubicado en el segundo lugar a nivel Latinoamérica y tasas de pobreza que superan el $35 \%$ de su población según el Foro Económico Mundial en sus reportes del 2017.

Otra falencia que se puede percibir de este programa que se ha evidenciado en su desarrollo, es que no fomenta de forma decidida la construcción de criterio político y cívico del ciudadano con pensamiento crítico, que vaya más allá del reconocimiento de las normas, que estén en capacidad de sobreponer el interés colectivo sobre el particular. De esta forma se identifican grandes retos que deben ser abordados en debates profundos par las entidades públicas y la sociedad civil frente a las nuevas generaciones y sus procesos de formación transformación que debe ser mediada por la educación.

El reto se centra en que el desarrollo de competencias ciudadanas mejore sustancialmente, la convivencia y participación de las personas desde el autoconocimiento en los entornos democráticos garantizando un proceso de educación integral con calidad, apoyado en el fortalecimiento de las competencias integradores en relación con la comunicación asertiva, el fomento al respeto por el arraigo cultural y la generación de vínculos de respeto entre personas con comportamiento cultural, aspecto o raíces diferentes, lo que conlleva a relaciones más exitosas y por ende al generación de redes asociativas que promueven el bienestar comunitario.

De esta forma, se parte de reconocer en la educación características como la integralidad y la calidad, las cuales inciden como base de la trasformación social que permite el desarrollo 
de competencias ciudadanas e transversales que permeen el actuar del sujeto en entornos sociales en función de logros multidimensionales.

Si la cultura democrática implica formar individuos con capacidad para tramitar sus intereses en una experiencia comunicada y compartida, las vivencias cotidianas de niñas, niños, maestros, maestras y en general de la comunidad, deberían ser retomadas y analizadas críticamente por la institución educativa. A la institución educativa le compete la formación de la personalidad básica del estudiante y la identidad es un elemento central de ella. (Alvarado \& Mieles, 2012, pág. 73) [1].

Finalmente, es de resaltar que el fortalecimiento y desarrollo de competencias ciudadanas se ha concentrado principalmente en la educación básica y media como resultado del programa planteado por el Ministerio de Educación Nacional en Colombia, Sin embargo, no se ha establecido un lineamiento definido propiamente dicho para educación superior, lo que ha conllevado a que cada institución de educación superior (IES), aborde las competencias ciudadanas en relación con su estudio análisis y apropiación en diferentes niveles de interés, lo que conlleva a que no exista un estado del arte planificado en la materia y cada institución las evidencie desde sus capacidades propias e interés particular.

\section{Métodos}

Se realizó una investigación de tipo descriptivo con enfoque de análisis primordialmente cuantitativo donde se abordó una muestra de 423 estudiantes de la Universidad ECCI de diferentes programas de formación frente a un universo aproximado de 18.000 estudiantes matriculados en el I periodo lectivo de 2018., se calculó una diversidad del universo de un $50 \%$ con un margen de error del $5 \%$ y un nivel de confianza del $95 \%$.

Dicha muestra se abordó por medio de un instrumento de recolección estructurado que tuvo como base conceptual el documento de medición de competencias ciudadanas, (ICFES, 2016) [7]. Sin embargo, los ítems se diseñaron frente a las características de la educación superior. El instrumento se diseñó en seis secciones que responden inicialmente a una información básica del encuestado, en segundo lugar se abordan preguntas sobre competencias integradoras, en tercer lugar se plantean ítems sobre competencias cognitivas y pensamiento crítico, posteriormente se analizan competencias emocionales y empatía, sucesivamente se aborda el tema de competencias civilidad y democracia y finalmente se indaga sobre asociatividad y redes. Para mayor conocimiento del instrumento se puede consultar en el siguiente vínculo: https://docs.google.com/forms/d/e/1FAIpQLSfpfBQp5fZWWo7pryOZknbiuGrz_tbTTFhze EGPVWE $\mathrm{paQ} 3 \mathrm{dg} /$ viewform? $\mathrm{usp}=$ sf_link.

\section{Resultados}

La base contiene un total de 423 personas encuestadas, se evaluaron un total de 97 variables, donde 5 variables corresponden a información sociodemográfica y de nivel educativo y las restantes 92 variables corresponden al estudio de las competencias ciudadanas. 


\subsection{Información sociodemográfica:}

A continuación, en la table 1 se muestra la distribución de la información sociodemográfica de las 423 personas encuestadas, en donde El 52\% son mujeres, el 47.8\% son hombres y el $2 \%$ no se identificó con ninguno de los dos generos de la encuesta.

Así mismo, la edad promedio de las personas encuestadas es de 24 años, la edad mínima dentro del estudio es de 16 años y la edad máxima es de 65 años.

Tabla 1. Datos sociodemográficos de la población

\begin{tabular}{|c|c|c|c|c|c|c|}
\hline \multicolumn{4}{|c|}{ Género } & \multicolumn{3}{|c|}{ Estadísticos } \\
\hline & & Frecuencia & Porcentaje & \multirow{2}{*}{\multicolumn{3}{|c|}{ Escriba su edad ( entre 16 y 50 ) }} \\
\hline \multirow[t]{6}{*}{ Válidos } & Hombre & 202 & 47,8 & & Válidos & 423 \\
\hline & Mujer & 220 & 52,0 & & Perdidos & 0 \\
\hline & Otro & 1 & 2 & Media & & 24,222 \\
\hline & Total & 423 & 100,0 & Mediana & & 22,000 \\
\hline & & & & Mínimo & & 16,0 \\
\hline & & & & Máximo & & 65,0 \\
\hline
\end{tabular}

Fuente: Cabrera, M (2016)

\subsubsection{Asociaciones más fuertes: preguntas competencias ciudadanas:}

Se describen las asociaciones más fuertes que se encontraron en las preguntas relacionadas a las competencias ciudadanas.

Tabla 2. Creencias religiosas - Nivel socio económico

\begin{tabular}{|c|c|c|c|c|c|c|c|}
\hline \multirow{8}{*}{$\begin{array}{c}\text { ¿En que nivel } \\
\text { soy tolerante } \\
\text { frente a las } \\
\text { siguientes } \\
\text { diferencias? } \\
\text { [Creencia } \\
\text { religiosa ] }\end{array}$} & \multicolumn{7}{|c|}{ ¿En que nivel soy tolerante frente a las siguientes diferencias? [Nivel socio económico ] } \\
\hline & Respuesta & 1. Muy bajo & 2. Bajo & 3. Medio & 4. Alto & 5. Muy alto & Total general \\
\hline & 1. Muy bajo & 3 & 3 & 3 & 2 & 3 & 14 \\
\hline & 2. Bajo & 1 & 13 & 12 & 7 & 3 & 36 \\
\hline & 3. Medio & 0 & 13 & 35 & 21 & 16 & 85 \\
\hline & 4. Alto & 0 & 4 & 7 & 91 & 39 & 141 \\
\hline & 5. Muy alto & 0 & 0 & 4 & 20 & 123 & 147 \\
\hline & Total general & 4 & 33 & 61 & 141 & 184 & 423 \\
\hline
\end{tabular}

Se observa que el $65 \%$ de los encuestados tienen un nivel alto de tolerancia frente a las diferentes creencias religiosas y la diferencia en el nivel socioeconómico. 
Tabla 3. Diferencias étnicas - Nivel educativo

\begin{tabular}{|c|c|c|c|c|c|c|c|}
\hline \multirow{8}{*}{$\begin{array}{c}\text { ¿En que nive } \\
\text { soy tolerante } \\
\text { frente a las } \\
\text { siguientes } \\
\text { diferencias? } \\
\text { [Diferencias } \\
\text { étnicas ] }\end{array}$} & \multicolumn{7}{|c|}{ ¿En que nivel soy tolerante frente a las siguientes diferencias? [Nivel educativo ] } \\
\hline & Respuesta & 1. Muy bajo & 2. Bajo & 3. Medio & 4. Alto & 5. Muy alto & Total general \\
\hline & 1. Muy bajo & 3 & 1 & 1 & 0 & 2 & 7 \\
\hline & 2. Bajo & 1 & 12 & 9 & 4 & 3 & 29 \\
\hline & 3. Medio & 1 & 9 & 23 & 14 & 3 & 50 \\
\hline & 4. Alto & 0 & 3 & 12 & 95 & 24 & 134 \\
\hline & 5. Muy alto & 1 & 0 & 9 & 25 & 168 & 203 \\
\hline & Total genera & 6 & 25 & 54 & 138 & 200 & 423 \\
\hline
\end{tabular}

Fuente: Cabrera, M (2016)

El 75\% de los encuestados también presentan un nivel de tolerancia muy alto cuando se evalúa el nivel de tolerancia de la diferencia étnica con la diferencia en el nivel educativo.

Tabla 4. Afinidad política - Nivel socio económico

\begin{tabular}{|c|c|c|c|c|c|c|c|}
\hline \multirow{2}{*}{$\begin{array}{c}\text { ¿En que nivel } \\
\text { soy tolerante }\end{array}$} & \multicolumn{2}{|c|}{ ¿En que nivel soy tolerante frente a las siguientes diferencias? [Nivel socio económico ] } \\
\cline { 2 - 9 } frente a las & Respuesta & 1. Muy bajo & 2. Bajo & 3. Medio & 4. Alto & 5. Muy alto & Total general \\
\cline { 2 - 9 } $\begin{array}{c}\text { siguientes } \\
\text { diferencias? }\end{array}$ & 1. Muy bajo & 3 & 0 & 2 & 1 & 2 & 8 \\
\cline { 2 - 9 }$\left[\begin{array}{c}\text { [Afinidad } \\
\text { politica }]\end{array}\right.$ & 2. Bajo & 0 & 12 & 10 & 8 & 8 & 38 \\
\cline { 2 - 9 } & 3. Medio & 1 & 10 & 31 & 50 & 33 & 125 \\
\cline { 2 - 9 } & 4. Alto & 0 & 10 & 15 & 72 & 65 & 162 \\
\cline { 2 - 9 } & Total general & 0 & 1 & 3 & 10 & 76 & 90 \\
\hline
\end{tabular}

En cambio cuando se evalúa el mismo nivel de tolerancia del nivel socio económico con respecto el nivel de tolerancia según su afinidad política, se observa que solo el 53\% de las personas presentan un nivel de tolerancia alto.

Tabla 5. Afinidad política - Nivel educativo

\begin{tabular}{|c|c|c|c|c|c|c|c|}
\hline \multirow{2}{*}{ ¿En que nivel } & \multicolumn{7}{|c|}{ ¿En que nivel soy tolerante frente a las siguientes diferencias? [Nivel educativo ] } \\
\hline & Respuesta & 1. Muy bajo & 2. Bajo & 3. Medio & 4. Alto & 5. Muy alto & Total general \\
\hline Soy toielanite & 1. Muy bajo & 3 & 0 & 0 & 1 & 4 & 8 \\
\hline Trente a las & 2. Bajo & 2 & 14 & 8 & 6 & 8 & 38 \\
\hline Slguientes & 3. Medio & 0 & 2 & 32 & 52 & 39 & 125 \\
\hline Qunerenclas? & 4. Alto & 0 & 9 & 10 & 72 & 71 & 162 \\
\hline [Alinidad & 5. Muy alto & 1 & 0 & 4 & 7 & 78 & 90 \\
\hline & Total genera & 6 & 25 & 54 & 138 & 200 & 423 \\
\hline
\end{tabular}

De igual forma cuando se analiza el nivel de tolerancia de la afinidad política contra el nivel educativo, solo el $54 \%$ de los encuestados presentan niveles altos de tolerancia. 
Después de analizar las distintas variables asociadas al nivel de tolerancia, se puede decir que es evidente que el nivel de tolerancia de la afinidad política tiene un grado de asociación negativa frente a las diferencias evaluadas.

Tabla 6. Tolerancia y creencias religiosas - Participación en órganos de la institución

\begin{tabular}{|c|c|c|c|c|c|c|c|}
\hline \multirow{8}{*}{$\begin{array}{l}\text { ¿Participa o ha } \\
\text { participado en los } \\
\text { últimos tres años en los } \\
\text { siguientes grupos u } \\
\text { órganos colegiados: } \\
\text { [Universidad (órganos } \\
\text { de participación )] }\end{array}$} & \multicolumn{7}{|c|}{$\begin{array}{l}\text { ¿Qué nivel de transparencia percibe en la gestión de los siguientes grupos u órganos colegiados: [Universidad (órganos de } \\
\text { participación )] }\end{array}$} \\
\hline & Respuesta & 1. Muy bajo & 2. Bajo & 3. Medio & 4. Alto & 5. Muy alto & Total general \\
\hline & 1. Nunca participaria & 1 & 5 & 16 & 7 & 3 & 32 \\
\hline & 3. Nunca he participado & 2 & 21 & 62 & 68 & 5 & 158 \\
\hline & 2. Me gustaria participar pero no se como & 3 & 20 & 29 & 27 & 3 & 82 \\
\hline & 4. He participado pero ya no participo & 1 & 9 & 22 & 41 & 12 & 85 \\
\hline & 5. Si participo actualmente & 1 & 1 & 19 & 37 & 8 & 66 \\
\hline & Total general & 8 & 56 & 148 & 180 & 31 & 423 \\
\hline
\end{tabular}

Cuando se analiza a los encuestados que nunca han participado y que nunca participarían en los grupos u órganos de la universidad, solo el $25 \%$ perciben un nivel de transparencia medio o bajo frente a la gestión que realizan los grupos en la universidad.

Tabla 7. Participación - percepción transparencia gestión pública

\begin{tabular}{|c|c|c|c|c|c|c|c|}
\hline \multirow{2}{*}{$\begin{array}{l}\text { ¿Participa o ha } \\
\text { participado en los }\end{array}$} & \multicolumn{7}{|c|}{$\begin{array}{l}\text { ¿Qué nivel de transparencia percibe en la gestión de los siguientes grupos u órganos colegiados: [Gestión pública del gobierno } \\
\text { nacional ] }\end{array}$} \\
\hline & Respuesta & 1. Muy bajo & 2. Bajo & 3. Medio & 4. Alto & 5. Muy alto & Total general \\
\hline últimos tres años en los & 1. Nunca participaria & 7 & 11 & 9 & 2 & 3 & 32 \\
\hline siguientes grupos u & 3. Nunca he participado & 30 & 59 & 54 & 13 & 2 & 158 \\
\hline órganos colegiados: & 2. Me gustaria participar pero no se como & 20 & 31 & 25 & 6 & 0 & 82 \\
\hline [Universidad (órganos & 4. He participado pero ya no participo & 20 & 19 & 28 & 15 & 3 & 85 \\
\hline de participación )] & 5. Si participo actualmente & 31 & 12 & 14 & 8 & 1 & 66 \\
\hline & Total general & 108 & 132 & 130 & 44 & 9 & 423 \\
\hline
\end{tabular}

Fuente: Cabrera, M (2016)

En cambio cuando se compara este mismo grupo de personas que nunca han participado o que nunca participarían en los grupos universitarios contra el nivel de transparencia en la gestión pública del gobierno nacional, el $40 \%$ de los encuestados perciben que el nivel de transparencia en la gestión es medio o bajo. De esta forma se puede suponer que los temas relacionados a la política o al gobierno nacional se perciben de manera negativa (porcentajes muy variables entre las tablas que se observaron).

Se construyeron 3 indicadores con el fin de reducir la información de las 92 variables y poder analizar la correlación entre estos 3 indicadores: 
Percepción social: mide la confianza en los medios de comunicación, en la empatía a los diferentes actores sociales (funcionarios públicos, religiosos, políticos, etc.), a la tolerancia frente a diferentes ámbitos sociales creencias, afinidad política, nivel educativo, etc. Y la transparencia a diferentes grupos u órganos colegiados.

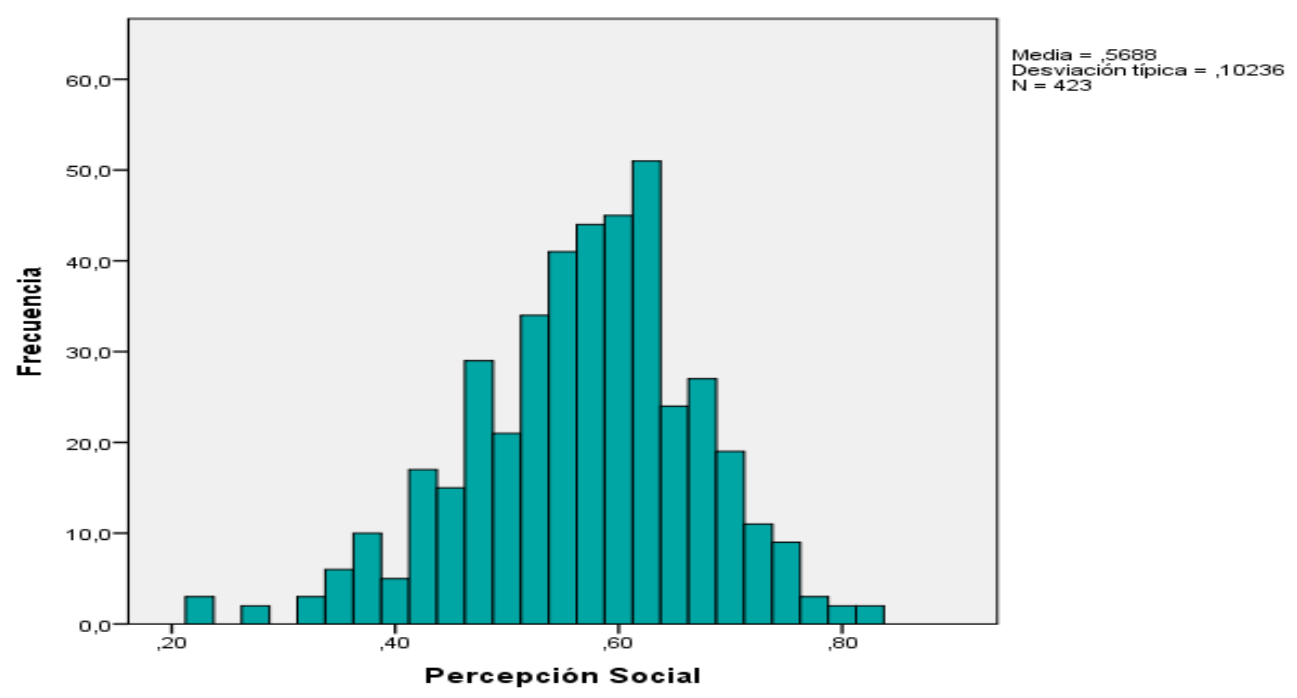

Figura 1: Percepción social

Participación social: mide el nivel de pertenencia a los diferentes escenarios sociales (barrio, universidad, ciudad, etc.), en la fomentación del dialogo para la solución de conflictos y en el conocimiento de las mediaciones de participación ciudadana en los diferentes entornos sociales .universidad, sindicatos, juntas, etc.

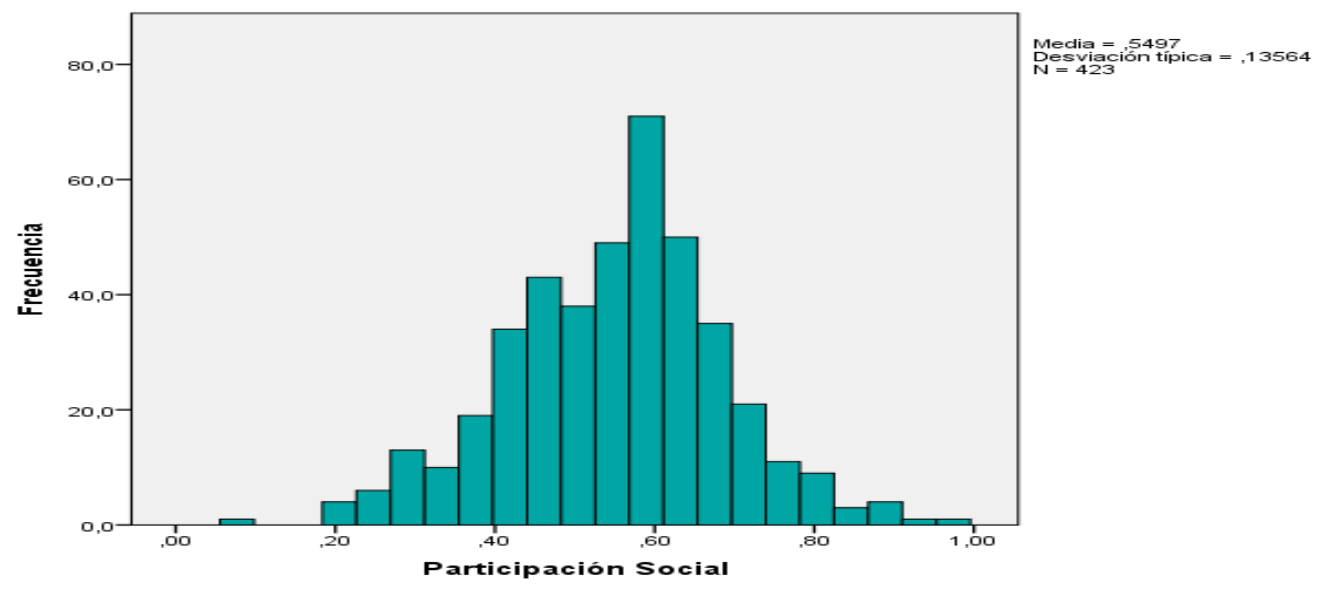

Figura 2: Participación social 
Relevancia social: mide la importancia de estar en las redes a nivel de desarrollo personal, familiar, laboral, social y cívico y también la importancia en la formación en competencias ciudadanas.

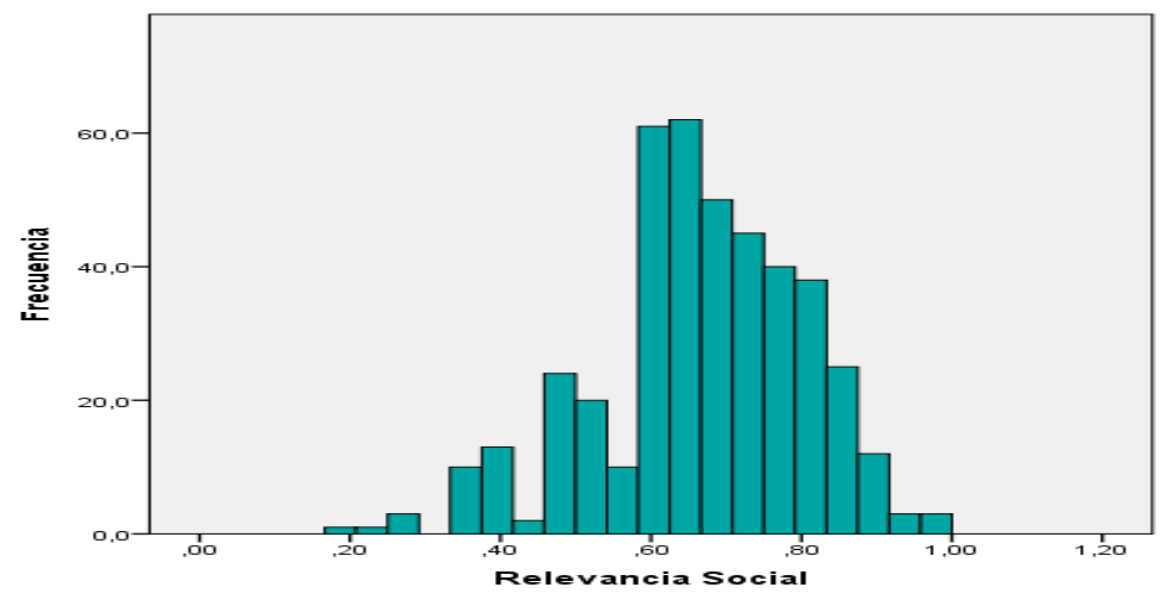
Media $=, 676$
Mesviacion tipica $=.13961$
$\mathrm{~N}=423$

Figura 3: Relevancia social

\section{Conclusiones}

Las competencias ciudadanas son relevantes para el desarrollo social de un territorio, en el caso colombiano se han venido trabajando articuladas con la educación media y básica en los últimos años sin embargo, en educación superior, se abordan de forma particular por cada institución, en el estudio realizado se evidencio que aunque los estudiantes reconocen la importancia de la tolerancia como factor base para la construcción de un mejor entorno social no se estima como un valor con alto grado de reconocimiento.

En términos generales se manejan unos rangos medios y medios altos de confianza, reconocimiento de la gestión, sin embargo, la tendencia cambia frente a posibles escenarios de participación como ciudadano, es decir, existe un desinterés por la participación cívica en función del interés colectivo.

El gran reto de la educación superior es aportar a la construcción de ciudadanía de una forma más decidida y contundente, fortaleciendo sus currículos en competencias ciudadanas, que posibiliten una mayor consciencia del estudiante en función de su rol en la sociedad.

Las grandes transformaciones que demanda Colombia, no pueden ser ajenas a la búsqueda permanente de sinergias de diversos actores que conlleven a la generación de una sociedad más cohesionada menos atomizada y que promueva la generación de desarrollo basada en el respeto e inclusión, lo que potencialice mayores y mejores oportunidades que fortalezcan e incrementen el capital social comunitario. 


\section{Referencias}

ÁlVAREZ, A., SANDOVAL, O., \& PUELLO, M. (2018). Estrategias pedagógicas para desarrollar competencias ciudadanas en estudiantes de derecho. Espacios (vol. 39 No 29), 1 - 9.

CHAUX, E., LLERAS, J., \& VELASQUEZ, A. (2004). Competencias ciudadanas: De los Estándares al Aula. 2004: Uniandes. Bogotá, Colombia.

Instituto Colombiano de Fomento para la Educación Superior ICFES, (2016), Módulo de Competencias ciudadanas Saber Pro 2016-2, Bogotá, Colombia.

KALDOR, M. (2005). La sociedad civil. Barcelona: 18 kriterios.

MIELES BARRERA, M., \& ALVARADO SALGADO, S. (2012). Ciudadanias y competencias ciudadanas. Estudios Políticos, 40, Instituto de Estudios Políticos, Universidad de Antioquia, (pp. 5375).

Ministerio de Educación Nacional, (MEN) 2006. Al tablero, Colombia, https://www.mineducacion.gov.co/1621/article-107411. Html revisado 30/11/2018)

Ministerio de Educación Nacional. (2006). Estándares Básicos de Competencias en Lenguaje, Matemáticas, Ciencias y Ciudadanas. Bogotá: Ministerio de Educación Nacional.

RAWLS, John (1995). Teoría de la Justicia. Ed. Fondo de Cultura Económica. Madrid. España.

RAWLS, John (2006). Liberalismo político. Traducido por Sergio Rene Madero. Ed. Crítica. Barcelona. España.

ROBISON, L., SILES M. \& SCHMID, A. (2003). El capital social y la reducción de la pobreza: hacia un paradigma maduro. En R. ATRIA Y M.SILES, (eds.), Capital social y reducción de la pobreza en América Latina y el Caribe: en busca de un Nuevo paradigma. Santiago de Chile: CEPAL-Michigan State University.

WOOLCOCK, M., \& NARAYAN, D. (2001). Capital social: Implicaciones para la teoría, la investigación y las políticas sobre desarrollo. 